\title{
Measurement of spallation cross sections for the production of terbium radioisotopes for medical applications from tantalum targets
}

\author{
H. Verhoeven ${ }^{\mathrm{a}}$, T.E. Cocolios ${ }^{\mathrm{a}, *}$, K. Dockx ${ }^{\mathrm{a}}$, G.J. Farooq-Smith ${ }^{\mathrm{a}}$, O. Felden ${ }^{\mathrm{b}}$, R. Formento-Cavaierc,d \\ R. Gebel ${ }^{\mathrm{b}}$, U. Köster ${ }^{\mathrm{e}}$, B. Neumaier ${ }^{\mathrm{b}}$, B. Scholten ${ }^{\mathrm{b}}$, I. Spahn ${ }^{\mathrm{b}}$, S. Spellerberg ${ }^{\mathrm{b}}$, M.E. Stamatia ${ }^{\mathrm{a}, \mathrm{f}}$, \\ S. Stegemann ${ }^{\mathrm{a}}$ \\ ${ }^{a}$ KU Leuven, Institute for Nuclear and Radiation Physics, Celestijnenlaan 200D, 3001 Heverlee, Belgium \\ ${ }^{b}$ Forschungszentrum Jülich GmbH, Wilhelm-Johnen-Strasse, 52428 Jülich, Germany \\ ${ }^{c}$ ARRONAX, 1 rue Arronax, 44800 Nantes, France \\ ${ }^{d}$ Advanced Accelerator Applications, a Novartis company, 20 Rue Diesel, 01630 Saint-Genis-Pouilly, France \\ ${ }^{e}$ Institut Laue-Langevin, 71 avenue des Martyrs, 38000 Grenoble, France \\ ${ }^{f}$ University of Ioannina, Physics Department, BP 1186, 45110 Ioannina, Greece
}

\begin{abstract}
We present new measurements of cumulative cross sections for the production of ${ }^{139} \mathrm{Ce}$ and ${ }^{149} \mathrm{~Tb}$ by protoninduced spallation of tantalum foil targets at different proton energies between 300 and $1700 \mathrm{MeV}$, using the COSY synchrotron at FZ Jülich. Those measurements are presented in the context of the production of medically-relevant radioisotopes for medical applications.
\end{abstract}

Keywords: spallation, terbium, cumulative cross section, tantalum

\section{Introduction}

Nuclear medicine is an important branch of medicine where radiopharmaceuticals are employed to target specific metabolic actions or specific cell types in the body for diagnostics or therapy. When a single biomolecule is coupled with different radioisotopes to interchangeably perform therapy and diagnostics, then a so-called theranostics approach is possible, allowing a more personalised care for the patient $[1]$.

In the last few years, a quadruplet of terbium isotopes has been identified for their high potential for theranostics: ${ }^{149} \mathrm{~Tb}$ for $\alpha$ therapy and PET imaging, ${ }^{152} \mathrm{~Tb}$ for PET imaging, ${ }^{155} \mathrm{~Tb}$ for SPECT imaging, and ${ }^{161} \mathrm{~Tb}$ for $\beta^{-}$therapy, Auger therapy, and

\footnotetext{
* Corresponding author

Email address: thomas.cocolios@kuleuven.be (T.E. Cocolios)
}

SPECT imaging [1]. An experimental programme is currently underway using neutron-deficient isotopes provided from CERN ISOLDE $[2,3]$ and more recently from CERN MEDICIS [4], while ${ }^{161} \mathrm{~Tb}$ is readily available from neutron-irradiation facilities such as the ILL in Grenoble (France). The production method at ISOLDE and MEDICIS relies on the spallation of tantalum foils induced by $1.4 \mathrm{GeV}$ protons from the CERN PSBooster. However, isobaric contamination induced by direct isobars or by pseudoisobaric molecules (e.g. ${ }^{155} \mathrm{~Tb}$ vs. ${ }^{139} \mathrm{Ce}^{16} \mathrm{O}$ ) might be collected as well $[2,5]$.

Furthermore, while the production rates at these facilities are sufficient to sustain the local and European demand for pre-clinical research, they are not suited for large-scale production for advanced clinical trials and, later, treatment. Different approaches are currently under investigation on how to upscale the production, including proton-induced spallation 
of Ta followed by mass separation, but with a higher primary beam intensity and optimised operational conditions. An important parameter that determines the production rates is the cross section. The spallation of Ta has been studied for various applications at different energies in the past $[6,7,8,9]$, however discrepancies by a factor 2 remain between the different measurements in the case of certain $\mathrm{Tb}$ isotopes that may impact future projects relying on those cross sections.

\section{Experimental method}

In order to resolve these discrepancies, we have performed a study of the cumulative cross sections for the production of radioisotopes in the proton-induced spallation of Ta at the COSY accelerator facility in FZ Jülich (Germany) [10]. Proton beams with energies ranging from $0.3 \mathrm{GeV}$ to $1.7 \mathrm{GeV}$, and intensity of the order of $100 \mathrm{pA}$ were used to irradiate stacks of $\mathrm{Ta}$ (measurement) and $\mathrm{Al}$ (normalisation) foils. The stacks were composed of Ta foils with thickness of $12,25,25,2,6,10,25 \mu \mathrm{m}$ in this order, followed by Al foils with thickness 50, 50, $50 \mu \mathrm{m}$. Spallation recoil losses for products exiting a foil are mitigated by the recoil capture from the preceding foil. The first and last foils of each material were systematically discarded due to an imbalance of these processes.

Each stack was irradiated once for a period of 2 to 5 hours at a fixed energy. The stacks were then retrieved from the irradiation point, separated, and the foils of interest were distributed to different measurement stations. The Al normalisation foils and the $25-\mu \mathrm{m}$ Ta foils were studied by $\gamma$-ray spectroscopy with one of four setups featuring a single coaxial highpurity germanium (HPGe) detector. The 2- and 6$\mu \mathrm{m}$ Ta foils were studied by $\alpha$-decay spectroscopy using a passivated implanted planar silicon (PIPS) detector. The total processing and transport time to the start of the measurement was of the order of 30 minutes, from which the only $\alpha$-emitting isotope with a measurable activity was ${ }^{149} \mathrm{~Tb}$. A typical $\alpha$-decay energy spectrum is shown in Fig. 1. The total measurement time for each foil varied between 1 hour to 1 day. In the months that followed the irradiation, the

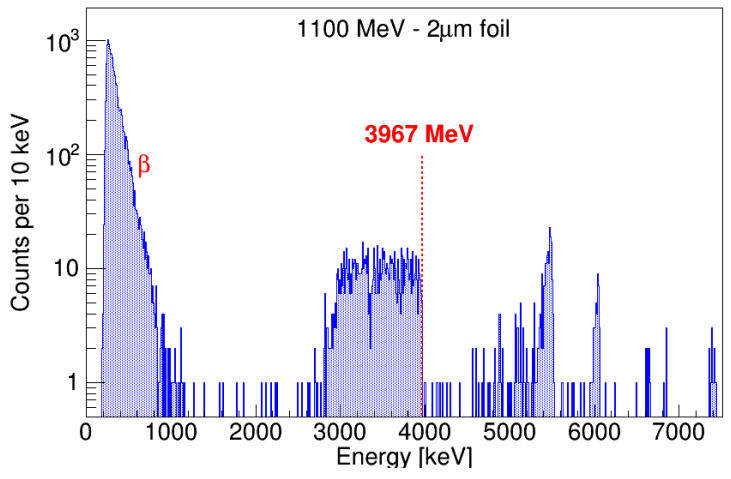

Figure 1: Alpha-decay energy spectrum from the $2-\mu \mathrm{m}$ foil irradiated at $1.1 \mathrm{GeV}$. The low-energy events are from $\beta$ particles, while the events from $5000 \mathrm{keV}$ upwards are background from self-sputtered residues from a ${ }^{241} \mathrm{Am}$ calibration source and other long-lived activity in the chamber. The $\alpha$-decay energy for ${ }^{149} \mathrm{~Tb}$ is marked at $3967 \mathrm{keV}$. The shape of the spectrum is well reproduced by the simulation of a homogeneous source distributed across $2 \mu \mathrm{m}$ of Ta.

2- and $6-\mu \mathrm{m}$ foils were also measured with an HPGe setup to study longer-lived activities.

\section{Analysis and discussion of the results}

The different spectra were analysed independently; the $\gamma$ rays and $\alpha$ particles were identified and linked with the decay of the respective isotope. The intensity of each transition was corrected for branching ratio and detector efficiency in order to determine the activity of each isotope at the time of measurement. Radioisotopes which share similar $\gamma$-ray energies had to be deconvoluted from each other.

Calculated foil activities at the time of measurement were extrapolated back to the time at which the proton irradiation ended (EOI). All cross sections presented in the following are cumulative cross sections since these are most relevant for production estimates at any facility. As a consequence, ${ }^{149 g} \mathrm{~Tb}$ measured 30 minutes after EOI or later includes feeding all short-lived precursors (e.g., ${ }^{149} \mathrm{Dy}, \mathrm{Ho}, \ldots \beta$ decay, or ${ }^{153} \mathrm{Ho}, \mathrm{Eu}, \ldots \alpha$ decay, and any decay chain leading to the isotope of interest), and ${ }^{139} \mathrm{Ce}$ activities derived from measurements several weeks after EOI 




Figure 2: Cumulative cross section for the production of ${ }^{149} \mathrm{~Tb}$ in the spallation of Ta. Two data points are shown for the 2 - and $6-\mu \mathrm{m}$ foils, in good intrinsic agreement. The data are compared to the literature data $[6,7,8]$. The operation energy for ISOL@MYRRHA and CERN ISOLDE are indicated with dashed lines and the effective energy range with the arrow.

include similar contributions (e.g., ${ }^{139} \mathrm{Pr}, \mathrm{Nd}, \ldots \beta$ decay). Additionally, decay corrections during the irradiation were taken into account, assuming constant irradiation. The activities from the Ta foils were normalised for the effective proton fluence with the activities of ${ }^{24} \mathrm{Na}$ in the $\mathrm{Al}$ monitor foils and their known spallation cross sections [11].

Details of the analysis can be found in Ref. [12]. A full table of results covering the entirety of this analysis will be published later. Here we present the results on ${ }^{149} \mathrm{~Tb}$ and ${ }^{139} \mathrm{Ce}$, which may appear as an isobar of ${ }^{155} \mathrm{~Tb}$ in the form of ${ }^{139} \mathrm{Ce}^{16} \mathrm{O}$.

In the case of ${ }^{149} \mathrm{~Tb}$, the cumulative cross section extracted with either the 2 - or the $6-\mu \mathrm{m}$ foils are in good agreement with each other, see Fig. 2. The values agree well with available literature data up to $1 \mathrm{GeV}$. Beyond that energy, two data sets are available and in disagreement $[6,8]$. The new data support the older data set from Winsberg [6]. Furthermore, it suggests the operation energy at CERN ISOLDE is not optimal as the cumulative cross section already decreases beyond $1.3 \mathrm{GeV}$. Meanwhile, a factor 2 can be gained between $0.6 \mathrm{GeV}$, the operational energy for the future ISOL@MYRRHA facility,

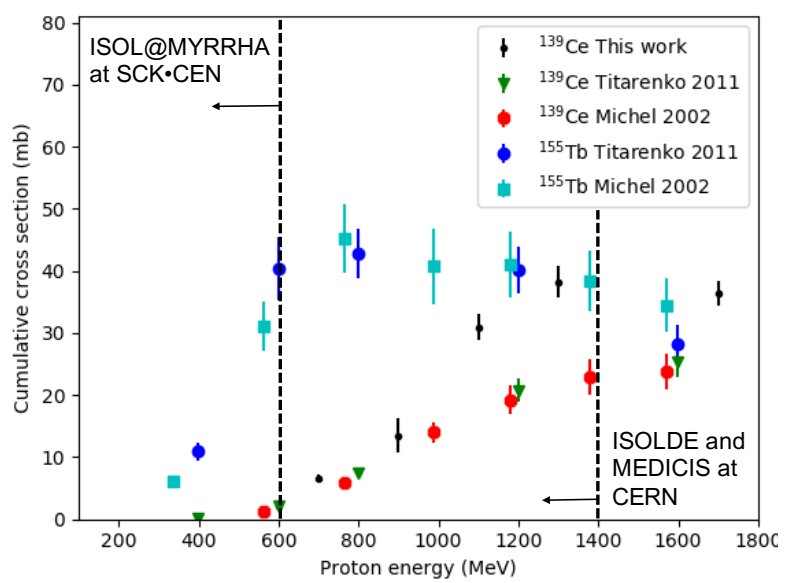

Figure 3: Cumulative cross section for the production of ${ }^{139} \mathrm{Ce}$ in the spallation of Ta from this work compared to literature data for the same isotope and for ${ }^{155} \mathrm{~Tb}[8,9]$. The operation energy for ISOL@MYRRHA and CERN ISOLDE are indicated with dashed lines and the effective energy range with the arrow. This comparison shows that the purity of the produced ${ }^{155} \mathrm{~Tb}$ sample can be increased by using a lower incident energy, such as foreseen at the ISOL@MYRRHA facility.

and $1 \mathrm{GeV}$, for the EURISOL facility.

A comparison can be made between the cumulative cross section for the production of ${ }^{155} \mathrm{~Tb}$ and ${ }^{139} \mathrm{Ce}^{16} \mathrm{O}$. Given that ${ }^{139} \mathrm{Ce}$ is much lighter than ${ }^{155} \mathrm{~Tb}$, more energy is required to boil off more nucleons in the spallation process, and the peak of the cross section is therefore shifted to a higher energy. As such, it can be seen from Fig. 3 that the energy at the ISOL@MYRRHA facility would be ideal to maximise the production of ${ }^{155} \mathrm{~Tb}$ while minimising the molecular isobaric contaminants.

\section{Conclusions and outlook}

A systematic study of the cumulative cross section of the proton-induced spallation of Ta has been performed at the COSY synchrotron in FZ Jülich at energies relevant for existing and future isotope separation online facilities. The results for ${ }^{139} \mathrm{Ce}$ and ${ }^{149} \mathrm{~Tb}$ have been presented in the context of the production of terbium radioisotopes for medical applications. The complete analysis of the radioisotopes 
measured in the range $A=100-180$ will be discussed in a forthcoming publication.

\section{Acknowledgements}

We would like to thank the COSY team for providing excellent beams for this research program.

This work was supported by FWO-Vlaanderen, a KU Leuven START grant, and a Marie SklodowskaCurie Innovative Training Network grant of the European Commission's Horizon 2020 Programme under contract number 642889 MEDICIS-PROMED.

\section{References}

[1] C. Müller, et al., J. Nucl. Med. 53 (2012) 1952 (2012).

[2] C. Müller, et al., Nucl. Med. Bio. 41 (2014) e58 (2014).

[3] C. Müller, et al., EJNMMI Radiopharm. Chem. 1 (2016) 5 (2016).

[4] R. M. dos Santos Augusto, et al., Appl. Sci. 4 (2014) 265 (2014).

[5] S. Kreim, et al., Nucl. Instrum. Meth. B 317 (2013) 492 (2013).

[6] L. Winsberg, Phys. Rev. 135 (1964) B1105 (1964).

[7] Y. T. Mironov, in: Conf. Nucl. Spec. Nucl. Structure, Sint-Petersburg (Russia), 2001, p. 276 (2001).

[8] Y. E. Titarenko, et al., Physi. At. Nucl. 74 (2011) 551 (2011).

[9] R. Michel, et al., J. Nucl. Sci. Tech. 39 (2002) 242 (2002).

[10] R. Maier, Nucl. Instrum. Meth. A 390 (1997) 1 (1997).

[11] Y. V. Aleksandrov, et al., in: Conf. Nucl. Spec. Nucl. Structure, Moscow (Russia), 1996, p. 223 (1996).
[12] H. Verhoeven, Master's thesis, KU Leuven, http://cds.cern.ch/record/2638456? $\ln =\mathrm{en}$ (2018). 